\title{
Modelling macroevolutionary patterns: An ecological perspective
}

\author{
Ricard V. Solé \\ 1 Complex Systems research Group, Department of Physics, FEN-UPC, \\ Campus Nord B4, 08034 Barcelona, Spain \\ 2 Santa Fe Institute, 1399 Hyde Park Road, Santa Fe, NM 87501, USA
}

\begin{abstract}
Complex ecosystems display well-defined macroscopic regularities suggesting that some generic dynamical rules operate at the ecosystem level where the relevance of the single-species features is rather weak. Most evolutionary theory deals with genes/species as the units of selection operating on populations. However, the role of ecological networks and external perturbations seems to be at least as important as microevolutionary events based on natural selection operating at the smallest levels. Here we review some of the recent theoretical approximations to ecosystem evolution based on network dynamics. It is suggested that the evolutionary dynamics of ecological networks underlie fundamental laws of ecology-level dynamics which naturally decouple micro from macroevolutionary dynamics. Using simple models of macroevolution, most of the available statistical information obtained from the fossil record is remarkably well reproduced and explained within a new theoretical framework.
\end{abstract}

\section{Macroevolution and extinction}

Looking at today's biosphere, it is hard to realize how much it has changed through millions of years of evolution. Some groups of organisms, once successful and ecologically dominant, went extinct. Extinction is the eventual fate of all species. Even for some of the most succesful groups that flourished over very long periods of time became extinct and vanished. Their remains are provided by the fossil record, an incomplete but rather informative data set [7]. As David Jablonski points out: "it is hard to resist the fossil record as a source of spectacular evolutionary triumphs, grotesqueries and catastrophes" [25]. From the Cambrian explosion of Metazoan life (about 550 million years ago) complex forms have evolved on land and sea and a pattern of increasing diversity (figure 1) is matched by a pattern of extinction punctuated by large-scale events of devastating consequences (figure 2).

Extinction has been seldom considered as a relevant ingredient in neodarwinian theories. The classical view suggested by Darwin involved a slow process of decline: "species and groups of species gradually disappear, one after another, first from one spot, then from another, and finally from the world". The rapid, sometimes massive extinction of entire groups was assumed to be due to the incompleteness of the record. But we certainly know that this is not the case: extinctions happen to occur at different intensities in different moments of life's history. The record shows many small events together with some few, mass 


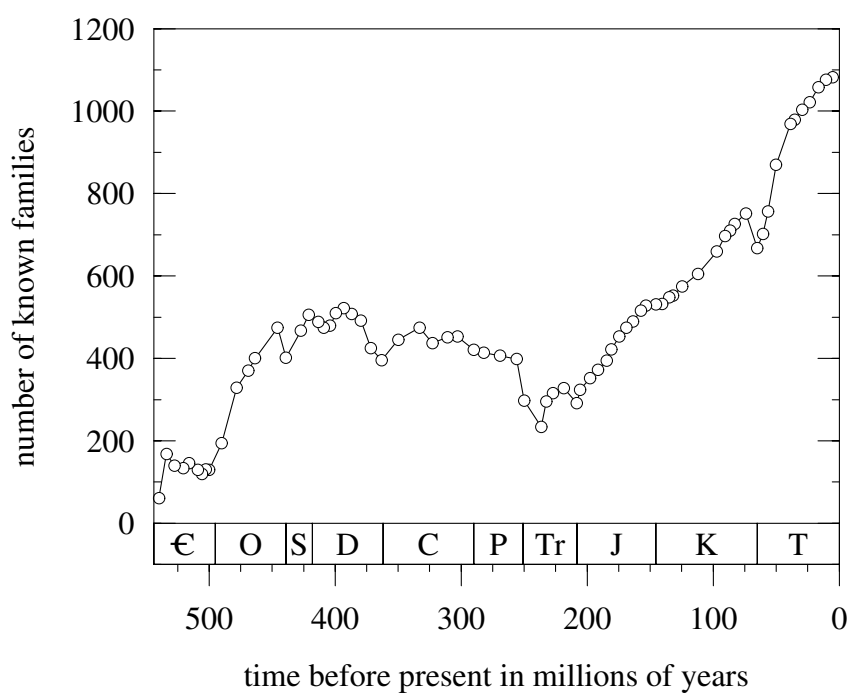

Fig. 1. Number of known marine families alive over the time interval from the Cambrian to the present. Data compiled by J. Sepkoski.

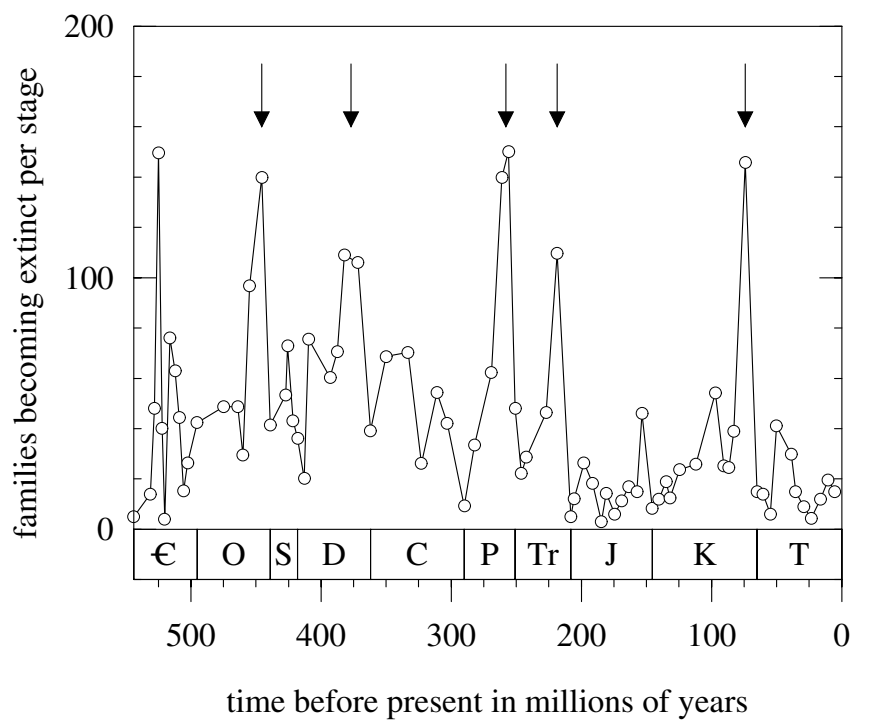

Fig. 2. Estimated extinction of marine animals in families per stratigraphic stage since the Cambrian. The arrows indicate the positions of the "big five" mass extinctions.

extinctions that wiped out a great part of Earth's diversity (see table 1). Of particular note are the five large peaks in extinction marked with arrows (figure 2). These are the "big five" mass extinction events which marked the ends of the Ordovician, Devonian, Permian, Triassic, and Cretaceous periods. 
Table 1. Extinction intensities at the genus and species level for the big five mass extinctions of the Phanerozoic. Estimates of genus extinction are obtained from directed analysis of the fossil record while species loss is inferred using a special statistical technique.

\begin{tabular}{|c|c|c|}
\hline & Genus loss (observed) & Species loss (estimated) \\
\hline End Ordovician & $60 \%$ & $85 \%$ \\
\hline Late Devonian & $57 \%$ & $83 \%$ \\
\hline Late Permian & $82 \%$ & $95 \%$ \\
\hline End Triassic & $53 \%$ & $80 \%$ \\
\hline End Cretaceous & $47 \%$ & $76 \%$ \\
\hline
\end{tabular}

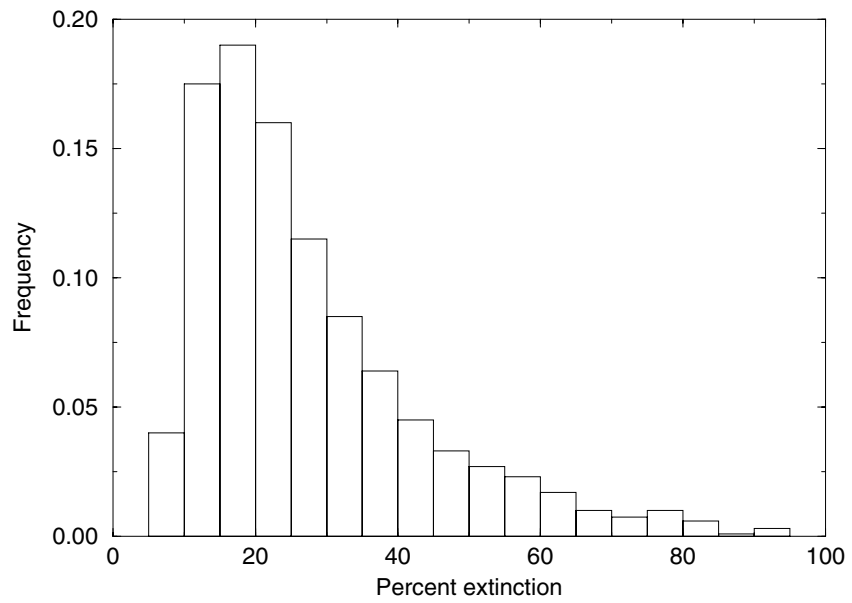

Fig. 3. Frequency distribution of extinction events. It shows a continuous range of values (instead of a bimodal one, as would be expected from a two-regime process, see text). We can see a maximum indicating a possible characteristic scale.

Although most neodarwinian theory almost ignores extinction, the fact is that the number of species extinctions in the history of life is almost the same as the number of originations 52 . Early analyses suggested that two basic regimes were involved in the overall pattern of extinction. The first would be "background extinctions" (possibly due to biological competition) and a second one, the "mass extinction" regime (perhaps associated to external stress). The observation of a continuous distribution (figure 3) does not support this view. Instead, it suggests a common causal origin for both large and small events. The problem is, of course, the nature of such an explanation.

Two major types of explanation have been suggested over the last decades. In one of them extinctions (particularly the large ones) result from external (non-biotic) events such as meteorite impacts, volcanic eruptions or changes in the magnetic field of Earth [52] [15] [50] [51]. This view has an obvious interest 
and relevance. There is clear evidence for external perturbations of the biosphere throughout the Phanerozoic and any theory of macroevolution should incorporate them. The end-Cretaceous event $(\mathrm{K} / \mathrm{T})$ is particularly well known and is consistent with a high-energy asteroid impact which generated severe darkening with a temporal cesation of photosynthetic activity on a very large scale and a rapid decrease in primary productivity (see below).

However, one should ask if these external events explain the previously mentioned features or are instead the trigger points of a cooperative biotic response. In this sense, it has been shown that the response of the biosphere to the size of the perturbation is far from linear [20] and the evidence does not suggest mass extinctions generally caused by such impacts. Actually a rather extensive search for extraterrestrial signatures at other stratigraphic intervals recording mass extinctions has been essentially negative 16. In fact some impact structures have no link with known extinction events. This is the case of the Montagnais impact structure, with a size of $45-\mathrm{Km}$ wide and an estimated age of $\approx 51$ million years, with no associated extinction event. And a potentially gigantic impact crater found in the Kalahari desert (with a diameter of $\approx 350 \mathrm{Km}$ ) has been dated around the Jurassic-Cretaceous boundary, were no evidence for severe extinctions is known.

Most published studies on externally-driven extinctions involve the analysis of available data together with a number of (usually qualitative) hypothesis concerning the correlations between physical and biotic patterns. Few theoretical models in the paleobiological literature have developped quantitative predictions of statistical patterns and in this sense their conclusions are mainly based in a priori assumptions of what mechanisms are at work. There are a few relevant properties of the fossil record that should be explained by any plausible theory of macroevolution 64]:

1. The Extinction pattern of species (or families or other taxonomic units) is clearly "punctuated" (strictly speaking this term is not properly used in the same context as it was first introduced in evolutionary biology). This means that rapid changes can be seen in the system in terms of large extinction (or diversification) events. This pattern has been shown to display long-range correlations 63] 65] 24.

2. The distribution of extinctions $N(m)$ of size $m$ follows a power-law decay with $N(m) \propto m^{-\alpha}$ with $\alpha \approx 2[62$ [41]

3. The lifetime distribution of family durations $N(t)$ follows a power-law decay $N(t) \propto t^{-\kappa}$ being $\kappa \approx 2[58]$.

4. The statistic structure of taxonomic systems also shows fractal properties. For example, the number of genus formed by $S$ species, $N_{g}(S)$, follows a power-law distribution with $N_{g}(S) \propto S^{-\alpha_{b}}$ with $S^{-\alpha_{b}} \approx 2$ [11] [12].

5. A study of the rates at which different groups of organisms go extinct through time shows that a species might disappear at any time, irrespective of how long it has already existed. This result, first reported by Leigh Van Valen strongly modified the ecological view of macroevolution [68] 8]. The fine-scale structure of these patterns is, however, episodic (see figure 4) and reflect the 


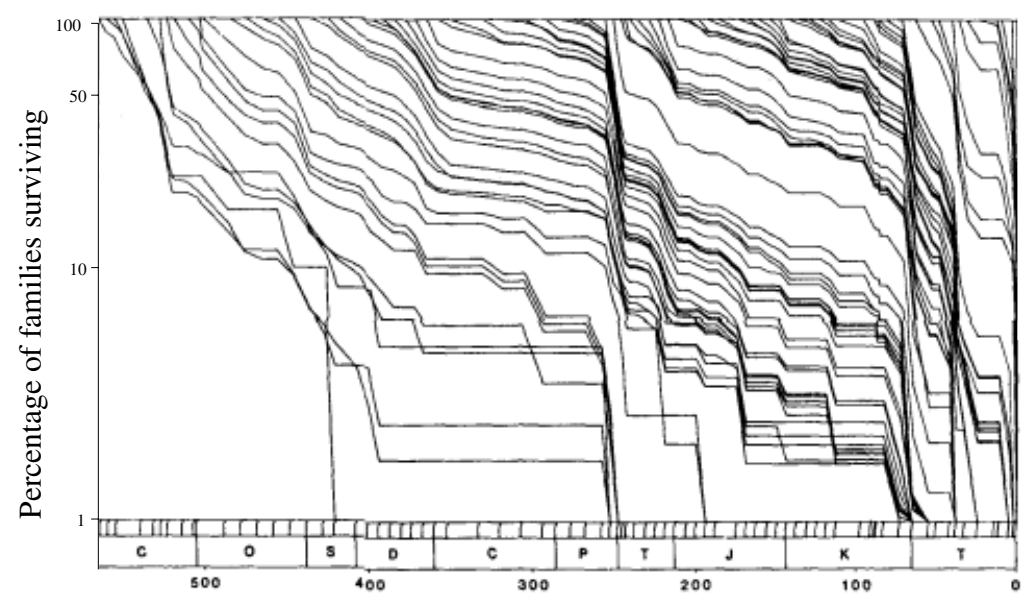

Millions of years ago

Fig. 4. Survivorship of 2316 families of marine animals over the past 600 Million years. Each line is a so called pseudocohort which starts (upper left) with th efamilies present in the fossil record at a point in time. Mass extinctions appear as sharp drops in survivorship (adapted from Raup, 1986).

interplay between slow dynamical processes and rapid changes that appear as drops in the diversity curves [54].

The presence of long-range correlations in the fossil record time series has been a source of controversy [43] 32. One clear conclusion from the disagreements between different studies is that the direct application of spectral techniques to the fossil record data has been far from appropriate, and the only clear conclusion is that there are long-range correlations [1], although their range and origins are debatable. Some authors have recently explored the problem of properly analysing the FR data sets [18] by means of the Lomb's method, which allows to obtain appropriate characterizations of uneven time series with nonstationary behavior. In this context, V. Dimri and M. Prakash have found evidence of long-range correlations together with a periodic component, thus confirming the presence of at least to types of structures in the large-scale dynamics of the biosphere. These results have been confirmed by means of wavelet analysis (Solé and Valverde, unpublished). The wavelet transform [49] replaces the Fourier transform's sinusoidal waves by a family generated by translations and dilations of a window called a wavelet. In this sense, a big disadvantage of a Fourier expansion is that it has only frequency resolution and no time resolution. This means that although we might be able to determine all the frequencies present in a signal, we do not know when they are present. The wavelet analysis takes advantage of nonstationary behavior and allows to see that the fossil record shows a fractal pattern over long time scales. 
More recently, Plotnick and Sepkposki have presented a re-analysis of the available data suggesting that it is better understood in terms of a conceptual model, based on a hierarchy of levels that interact in a multiplicative fashion 48. The authors compare their model outcomes with improved extinction and origination data and find a good agreement, thus concluding that it provides a better understanding of macroevolutionary patterns than the ones presented by previous models (to be discussed below). In this sense, it is important to establish appropriate criteria allowing to evaluate the value of a given model when compared to the fossil record data. Most of the published literature (both in paleontology and physics) present models or analysis that concentrate in one or two basic traits, completely ignoring the whole picture that emerges when all the available data is taken into account. Although there are many open questions emerging from the new theoretical approaches [39] 27] one clear test for any sensible theoretical explanation of macroevolutionary patterns of extinction and diversification is to be able to reproduce as many quantitative traits as possible.

A number of mathematical models of long-term evolutionary patterns have been proposed by several authors. The earliest and most appealing of them is Sepkoski's model of competition, which assumed that the Cambrian, Paleozoic and Modern evolutionary faunas each diversified logistically as a consequence of early (exponential) growth followed by a slowing down as ecosystems became filled [56] 26]. By tuning several parameters and a set of external perturbations similar to those suggested by the fossil record, a very similar pattern of diversification is obtained. Although this model is simple and appealing, the lack of a unique parameter determination and the number of assumptions implicit in the competition model makes it essentially descriptive. A similar criticism can be applied to other models, although their value as a theoretical framework is undeniable.

More recently, a new generation of models give support to a scenario where externally-driven ecological responses might play a relevant role [5] 64 [47. These models have shown that it is possible to reproduce many quantitative traits displayed by the fossil record and even a new theoretical interpretation of the macroevolutionary process. The implications for macroevolution are significant. They suggest that multispecies interactions are a key ingredient in shaping the structure of evolving ecosystems and that the fate of individual species would be the result of collective phenomena, not reducible to a list of independent fitnesses. In this context, it has been suggested that long-term, ecological-level network dynamics provides the natural decoupling between micro- and macroevolutionary patterns [59] 64].

Evolution does not take place in an ecological vacuum. Even the first ecologies emerging from the Cambrian boundary have been shown to display some of the characteristic features of modern ecologies [17]. Besides, the aftermath of mass extinctions show that ecological-based responses underlie the extraordinarily protracted lag-times for recovery before similar diversity levels are reached again 33] 21] 22] 23. Besides, in many well-documented cases, changes in the pattern of extinction and diversification are directly associated with ecolog- 
ical responses (such as the emergence and evolution of mineralized exoskeletons triggered by predation).

This review has been writen with a partisan view of macroevolution based on an ecological representation of evolving biological structures. In that sense, I am not considering other types of models where such a network of interactions is essentially ignored. This is certainly a limitation, since I am sure that other approaches, such as Newman's stress model [41] 42] or Sibani's reset model [57] have a very important value and are close to reality in many ways (not to mention the fact that in many ways the Phanerozoic involves different sources of innovation and thus of nonstationarity). In truth, the final answers to the problems arising from the patterns displayed by the fossil record are likely to be understood by using appropriate ingredients provided by these different approximations.

\section{Coevolution on a rugged fitness landscape}

The first attempt to understand large-scale evolution in terms of a complex adaptive system with interactions among different species was introduced by Kauffman and Johnsen [29], who used previous theoretical work on fitness landscapes [30]. The model is inspired in previous theoretical work by Per Bak and co-workers on self-organized criticality [3, [5], 6]. The basic idea of the fitness landscape metaphor is that single species can be characterized in terms of a string of genes or traits, $S_{1} S_{2} \ldots S_{N}$ which constitute the "genotype" and have an associated real number $\Phi\left(S_{1} S_{2} \ldots S_{N}\right) \geq 0$ usually normalized to one. This quantity is the fitness of the string and the distribution of fitness values over the space of genotypes defines the fitness landscape (figure 5). Depending upon the distribution of the fitness values, the fitness landscape can be more or less rugged. The rugeddness of the landscape is a crucial property, strongly constraining the dynamics. If we consider a population of strings, then the way this population evolves depends on how mountainous the landscape, how large is the population size and on mutation rates [30].

Rugged landscapes (RL) are a common feature of many different complex systems, from RNA viruses to glasses. They have been studied from various viewpoints in disparate areas such as biophysics of macromolecules to combinatorial optimization problems.

If we want to model macroevolutionary dynamics, then in principle many different, coupled species have to be taken into account. Each species is characterized by the number of traits $N$ and by another parameter $K$ which is in fact a measure of the degree of ruggedness (it gives the number of epistatic interactions among genes/traits). The fitness of a given string is obtained by means of a table of values, as the one shown in figure 6 . Here a $N=3, K=2$ system is shown, together with the corresponding landscape, here just a simple three-dimensional cube. Adaptive walks only occur in the direction of increasing $W$, and so the system is finally frozen at one of the two local maxima (here indicated by means of circles). 


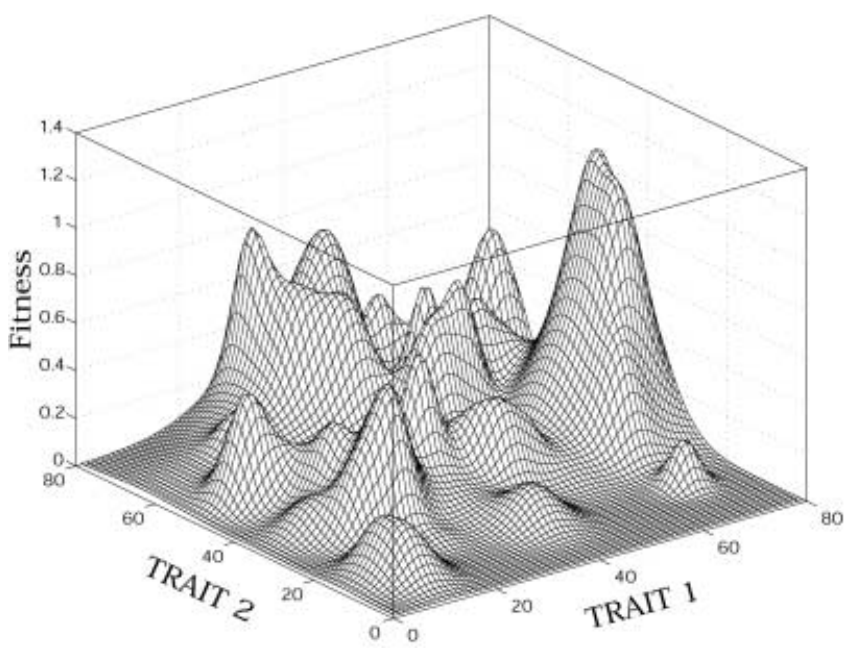

Fig. 5. Simple, two-dimensional continuous rugged landscape. This corresponds to the early metaphor suggested by Sewall Wright. Here a given species is defined as a twotrait pair $\left(x_{i}, y_{i}\right)$. For each pair a fitness $\phi\left(x_{i}, y_{i}\right)$ can be defined. The ruggeddness of the landscape controls the population flow through trait space. If the landscape is very rugged, historic effects play a dominant role.

K=2 input structure:
fitness table:
\begin{tabular}{|lll|l|l|l|l|l|}
\hline 1 & 2 & 3 & w1 & w2 & w3 & w \\
\hline 0 & 0 & 0 & 0.6 & 0.3 & 0.5 & 0.47 \\
0 & 0 & 1 & 0.1 & 0.5 & 0.9 & 0.50 \\
0 & 1 & 0 & 0.4 & 0.8 & 0.1 & 0.43 \\
0 & 1 & 1 & 0.3 & 0.5 & 0.8 & 0.53 \\
1 & 0 & 0 & 0.9 & 0.9 & 0.7 & 0.83 \\
1 & 0 & 1 & 0.7 & 0.2 & 0.3 & 0.40 \\
1 & 1 & 0 & 0.6 & 0.7 & 0.6 & 0.63 \\
1 & 1 & 1 & 0.7 & 0.9 & 0.5 & 0.70 \\
\hline
\end{tabular}

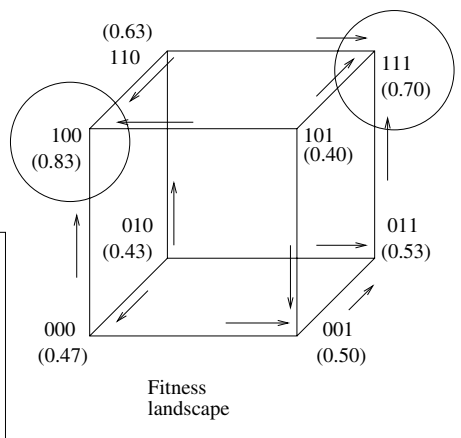

Fig. 6. How to built a fitness landscape. We have a $N=3$ string with $K=2$ interactions among each trait (so called epistatic interactions) and a table providing the fitness $W(i)$ of each individual trait given a particular string sequence.

This $N K$ model has been widely explored and many relevant results concerning its statistical properties have been derived. This is not surprising, given its close similarity with spin glass (SG) models. As in SG, frustration takes place and allows to understand the distribution of peaks when $K$ is tuned. The basic dynamics in this model involves adaptive walks. Here for a single species we choose a given trait and flip a coin (i. e. mutate) the bit. Then we look at the fitness table and if the average fitness of the new configuration is larger than the last one, an adaptive walk occurs and so a movement in the fitness landscape. 
If not, no walk is allowed to occur. This simple procedure leads to a hill climbing in the landscape until a local peak is reached. Afterwards, nothing happens. For $K=0$ (the Fujiyama landscape) no interactions among different traits are present and a very smooth landscape is obtained, with a single global maximum and an expected number of walks $L_{w}=N / 2$ to reach the optimum. This is a highly correlated, simple landscape.

At the other extreme, when $K=N-1$, the landscape is fully random. Several interesting properties have been reported, among others: (a) the number of local fitness optima is maximum; (b) the expected number of fitter one-mutant variants drops by $1 / 2$ at each improvement step; (c) the length of adaptive walks to optima are short, with a characteristic value $L_{w} \approx \log (N)$.

For an uncorrelated landscape, each bit string is assigned a fitness at random, so even single-bit changes may have very different fitness values. Although this is not a biologically realistic model, it allows to obtain some basic analytic results and will help to understand the coevolutionary patterns arising from the Kauffman-Johnsen model.

The number of maxima is easily calculated. If only one-bit mutations per string are allowed to occur, each string has $N$ one-bit neighbors. The probability that any one string has higher fitness than any of its neighbors is:

$$
P_{1}=\frac{1}{N+1}
$$

and thus the average number of local maxima is $\left\langle M_{1}\right\rangle=P_{1} 2^{N}$. The length of the walks can also be estimated [55. Let us assume that we start with the least fit string, and that the range of fitness values is constrained to the unit interval, with a uniform distribution over fitness space. Any mutation will give a fitness increase with an average value, for the first walk, of $\left\langle F_{1}\right\rangle=1 / 2$. The second walk will increase the fitness to an average $\left\langle F_{2}\right\rangle=3 / 4$ and after $k$ walks we will have:

$$
\left\langle F_{k}\right\rangle=1-\left(\frac{1}{2}\right)^{k}
$$

It is easy to show that the probability of a string not reaching a local maximum after $k$ walks is:

$$
P_{k}=\left(1-\frac{1}{2^{k}}\right)^{N-1}
$$

If we define $\omega_{k}=1-P_{k}$, the probability $\mathcal{L}_{k}$ that the walk will last through the $k$-th step is:

$$
\mathcal{L}_{k}=\prod_{r=1}^{k} \omega_{r}=\prod_{r=1}^{k}\left[\left(1-\frac{1}{2^{r}}\right)^{N-1}\right]
$$

Most walks will proceed until $\mathcal{L}_{k}<0.5$, i. e. until $\approx \log (N-1)$ steps.

Now the problem is how to obtain a more complete picture of an evolving system formed by many species in interaction. This can be done by using the so called NKC model [29]. The parameter $C$ introduces the number of couplings between species. Again each species is represented by just a string (instead of 
a population of individuals) which somehow defines the average characteristics (the phenotype) for that particular species. Now each trait receives "inputs" from $C$ other traits belonging to different species. These traits are chosen at random between the $S$ species.

The NKC model shows two well defined dynamical regimes (phases). These regimes are the high- $K$, chaotic phase, where changes in the ecosystem are always taking place (i. e. the system does not settle down in a number of local optima) and the low- $K$, ordered (frozen) phase where local optima are reached by all species (the so called Nash equilibria in economic theory). At the boundary separating the two phases, complex dynamics takes place. For a given $N$, if $C$ is small (below a given threshold) then the whole population evolves into a state where no further changes take place and all species are at Nash equilibria. However, after a critical point is reached, the dynamics becomes chaotic and no final steady state is obtained. Just at the boundary, species in a finite system reach local peaks but any small perturbation generates a coevolutionary avalanche of changes through the system. The distribution of these avalanches is a power law, as expected for a critical state. Kauffman and Johnsen mapped these avalanches into extinction events, suggesting that the number of changes in species is proportional to the extinction of less-fit variants. If this analogy is used then the obtained scaling relation for avalanches of $S$ changes is $N(s) \propto S^{-1}$, which does not agree with the value reported from the fossil record. However, a further version of this model (allowing evolution in the parameters) has been shown to self-organize to the critical state 31 with avalanches following the correct $\tau=-2$ exponent (figure 7 ). The final picture that emerges from this last model is that as species tune their own landscapes (by readjusting the ruggedness) they poise the entire ecosystem close to the critical boundary.

Some analytic work on the NKC model has been done by Per Bak and coworkers. If we restrict ourselves to the $K=N-1$ case and assume a large number of species, the existence of two well-defined phases in the $(N, C)$-plane can be derived [4. Let us assume that the fitness values are uniformly distributed in the interval $U=[0,1]$. Additionally, let us assume that instead of keeping the $C$ randomly chosen foreign genes that any species depends on, we exchange this "quenched" randomness for "annealed". This just means that the $C$ connections are randomly assigned at each time step. Finally, let us consider a very large ecosystem so that a probability density can be defined. Here $\rho_{M}(F, t)$ will be the fraction of species with fitness $F$ and $M$ less-fit 1-mutan neighbors at time t.

Bak et al. define a quantitative measure of the evolutionary activity in the system [4]. This quantity, $A(t)$, gives the probability that a change in a random gene leads to higher fitness (and therefore is accepted):

$$
A(t)=\sum_{M=0}^{N}\left(1-\frac{M}{N}\right) \int_{0}^{1} d F \rho_{M}(F ; t)
$$

where $1-M / N$ is the probability that the change of a single random unit leads to higher fitness. 


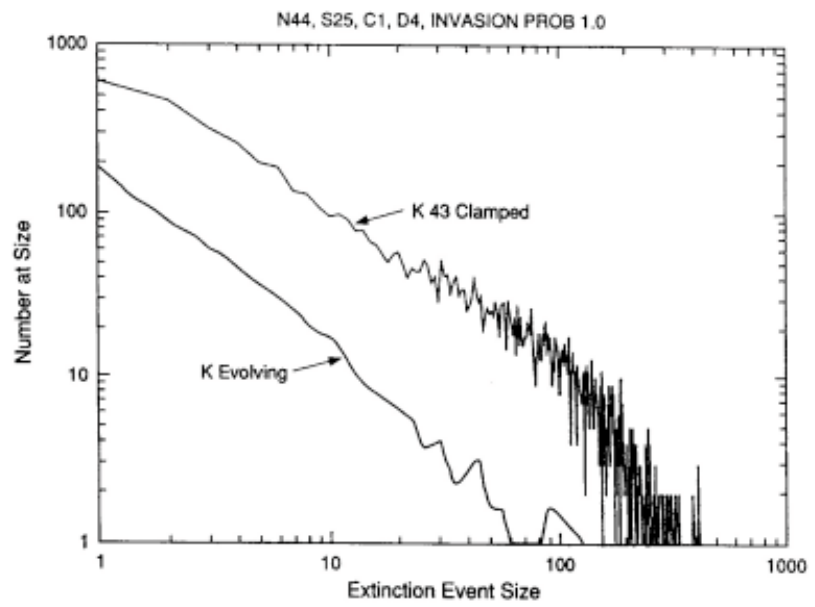

Fig. 7. Power law distribution of coevolutionary avalanches in the modified KauffmanJohnsen model. Here two different results are shown. One is for a NKC network where the $K$-parameter has been fixed to a high value (in the chaotic regime) and the lower one is obtained in a system where the landscape ruggedness is allowed to evolve. The parameters are $C=1, S=25, N=44$ and $K$ evolves towards an intermediate value $(K \approx 22)$.

Now the probability that such a mutation is accepted and leads to a fitness $F$ (for the changed species) is:

$$
\Phi(F ; t)=\int_{0}^{F} d F^{\prime} \phi\left(F^{\prime} ; t\right)
$$

where

$$
\phi\left(F^{\prime}, t\right)=\frac{1}{1-F^{\prime}} \sum_{M=0}^{N}\left(1-\frac{M}{N}\right) \rho_{M}(F ; t)
$$

Using these quantities, a master equation can be derived, leading to:

$$
\begin{aligned}
\frac{\partial}{\partial t} \rho_{M}(F, t)= & -\left(1-\frac{M}{N}\right) \rho_{M}(F ; t)+B_{M, N}(F) \Phi(F, t) \\
& -\frac{C}{N} A(t) \rho_{M, N}(F, t)+\frac{C}{N} A(t) B_{M, N}(F, t)
\end{aligned}
$$

This equation gives the time evolution of the (relative) number of species with fitness $F$ and $M$ less-fit neighbors. Here $B_{M, N}(F)$ is a binomial distribution with mean $F$, i. e.:

$$
B_{M, N}=\frac{N !}{M !(M-N) !} F^{M}(1-F)^{N-M}
$$

standing for the probability thet $M$ out of $N$ one-mutant neighbors to a genome with fitness $F$ are less fit than $F$. 
Although a detailed analysis of the parameter space can be derived, here only an estimation of this phase space will be obtained. A trivial solution of the master equation is given by all species placed in local fitness maxima:

$$
\rho_{M}^{*}(F, t)=\delta_{M, N} \rho(F)
$$

As usual, the relevance of this solution depends on the connectivity $C$. Here $\rho_{M}^{*}(F, t)$ will be attractive if $C=0$ and at the other extreme, when $C / N \ll 1$ we get

$$
\rho_{M}^{*}(F, t)=B_{M, N}(F)
$$

which corresponds to maximum activity $A=1 / 2$.

The interesting properties are observed at intermediate $(0<C<N)$ values of the connectivity. If some stationary activity is present for a given $C$ value, we could ask whether this activity stops or not. We can obtain an approximate relation between $C$ and $N$ that will give us the critical line separating frozen from chaotic phases.

We have previously mentioned that in NK landscapes with $K=N-1$, an average number of adaptive walks (until a local maximum is reached) follows a logarithmic dependence

$$
\mu_{1} \approx \ln (N)
$$

For a NKC landscape this is a lowest bound to the number of changes per species by which the NKC model can evolve to the fixed point $\rho_{M}^{*}(F, t)$.

Now, suppose that, for a given $C$-value, the species have been arranged in order to satisfy (10). A small perturbation is then introduced: the fitness of one species is changed to a random value, being the others in the same state. Such a perturbed species will need an average of $\mu_{1}$ steps before to reach a local maximum. But in fact the fitness of other species depends, through $C$, on the values taken by other genes/traits. If any of these genes/traits are among the $\mu_{1}$ ones that changed through the walk, the affected species will set back in evolution. Our question of course is whether or not the initial change can trigger a "chain reaction" able to percolate through the system. The critical condition is easily obtained:

$$
\mu_{1} \frac{C_{c r i t}}{N}=1
$$

i. e. when, on the average, one out of $C$ randomly chosen genes is among the $\mu_{1}$ changed genes. This gives the critical line in the $(N, C)$ space:

$$
C=\frac{N}{\ln (N)}
$$

This line separates the two phases.

\section{Network model of macroevolution}

One of the criticisms to the previous model (and other early models based on self-organized critical behavior, such as the Bak-Sneppen model [5]) is that they 
lack true extinction and diversification [35. Although the evolutionary activity in these models has something to do with the underlying extinction dynamics, it is not obvious how to map the first into the second. On the other hand, one of the obvious rules to be considered by any reasonable model is replacement of empty niches by surviving species. And these species will interact through a new, evolving network of connections.

The standard mathematical approach to population dynamics is the LotkaVolterra (LV) $n$-species model,

$$
\frac{d N_{i}}{d t}=N_{i}\left(\epsilon_{i}-\sum_{j=1}^{n} \gamma_{i j} N_{j}(t)\right)
$$

where $\left\{N_{i}\right\}, i=1, \ldots, n$ are the populations of each species. These models have been explored in deep. Two main qualitative problems have been considered: (i) small- $n$ problems, involving two or three species and (ii) large- $n$ models, involving a full network of interacting species.

The Lotka-Volterra equations used in most models of multispecies ecosystems are too difficult to manage if the matrix of interactions $\Gamma$ is formed by timedependent terms (as one would expect in an evolving ecosystem). We want to retain the basic qualitative approach, but our interest is shifted from population sizes to the appearance and extinction of species. Here species are defined as a binary variable: $S_{i}=0$ (extinct) or $S_{i}=1$ (alive). The state of such species evolves in time (now assumed discrete) according to

$$
S_{i}(t+1)=\Phi\left(\phi_{i}(t)\right)=\Phi\left(\sum_{j=1}^{n} \gamma_{i j}(t) S_{j}(t)\right)
$$

with $i=1, \ldots, N$. Here $\Phi(z)=1$ if $z>0$ and zero otherwise. Equation (2) can be understood as the discrete counterpart of (1), but involving a much larger

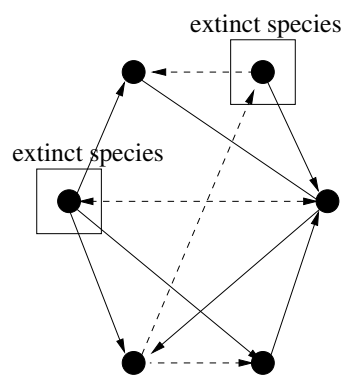

extinction

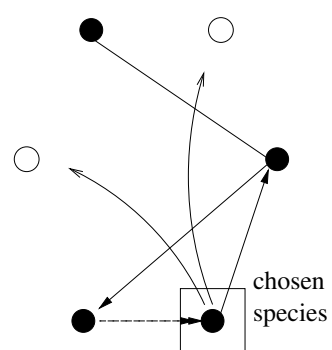

diversification

Fig. 8. Basic rules of the network model of evolution. After a number of species are extinct (here two of them), one of the survivors is chosen (center) to repopulate the network. The diversification is performed by copying the connections of the chosen species. These rules are completed with a random change of connections involving both internal and external changes. 
time scale. In this model, first introduced by Solé [59 60] 61], the $i-$ th species is in fact represented by the set of connections $\left\{\gamma_{i j}, \gamma_{j i}\right\}, \forall j$. The elements $\gamma_{i j}$ are the inputs and define how the the other species influence it. The symmetric elements $\gamma_{j i}$ are the outputs and represent the influence of this species over the remaining ones in the system.

The dynamics of this model is defined in three steps:

1. Changes in connectivity. Each time step we change one connection $\gamma_{i j}$ which takes a new, random value $\gamma_{i j}(t+1) \in[-1,1]$, for each $i=1, \ldots N$, with $j \in\{1, \ldots, N\}$ chosen at random. This rule is linked to changes in species interactions. They could be associated with external causes or simply be the result of small changes as a consequence of coevolution. This rule introduces random, small changes into the network.

2. Extinction. The local fields $\phi_{i}(t)=\sum_{j} \gamma_{i j}(t) S_{j}(t)$ are computed, and all species are synchronously updated following (2). If the $k-$ th species goes extinct, then all the connections that define it are set to zero, that is $\gamma_{k j}=$ $\gamma_{j k} \equiv 0, \forall j$. This updating introduces extinction and selection of species. Those sets of connections which make a species stable will remain. But in removing a given species, some positive connections, with a stabilizing effect on other species can also disappear, and the system can become more unstable.

3. Replacement. Some species are now extinct (i. e. $S_{k}=0$ ), empty sites are available and diversification is introduced. A living species is picked up at random and "copied" in the vacant niches. The new species are basically identical to the one randomly choosen, except for a small random change in all their connections. Specifically, let $S_{c}$ the copied species. For each extinct species $S_{j}$ (vacant spaces), the old connections are set to zero, and the new connections $\gamma_{i j}$ and $\gamma_{j i}$ are given by $\gamma_{k j}=\gamma_{c j}+\eta_{k j}$ and $\gamma_{j k}=\gamma_{j c}+\eta_{j k}$. Here $\eta$ is a small random variation (typically $\eta_{k j}=O\left(10^{-2}\right)$. In this way, the new species are the result of the diversification of one of the survivors.

The random changes in the network connections make the trophic links between species more and more complex. We can quantify such complexity by
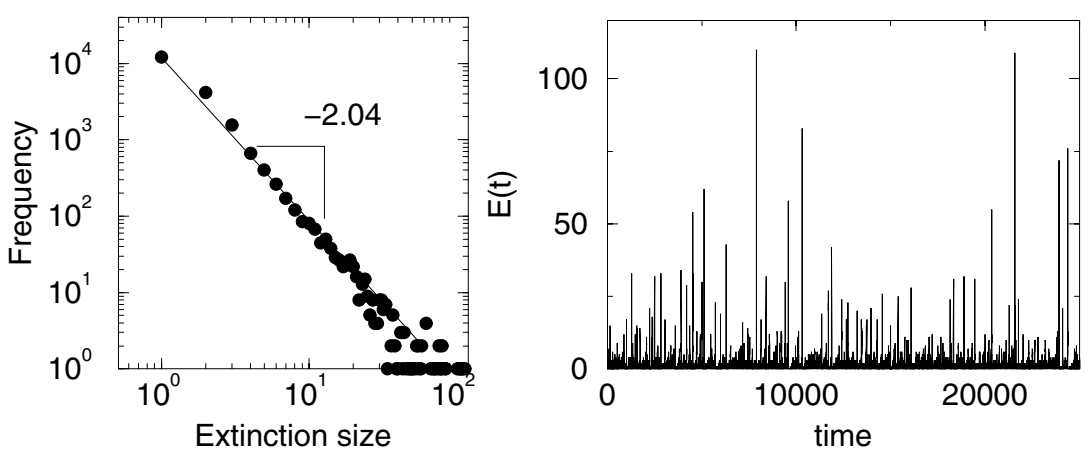

Fig. 9. Power law distribution of extinction events in the network model (left). The corresponding time series is shown (right). 
means of an adequate statistical measure. Let us first consider the time evolution of connections. Let $P\left(\gamma^{+}\right)$and $P\left(\gamma^{-}\right)=1-P\left(\gamma^{+}\right)$be the probability of positive and negative connections, respectively. The time evolution of $P\left(\gamma^{+}, t\right)$ is defined by the master equation

$$
\frac{\partial P\left(\gamma^{+}, t\right)}{\partial t}=P\left(\gamma^{-}, t\right) P\left(\gamma^{-} \rightarrow \gamma^{+}\right)-P\left(\gamma^{+}, t\right) P\left(\gamma^{+} \rightarrow \gamma^{-}\right)
$$

From the definition of the model, we have a transition rate per unit time given by $P\left(\gamma^{+} \rightarrow \gamma^{-}\right)=P\left(\gamma^{+} \rightarrow \gamma^{-}\right)=1 /(2 N)$ and so we have an exponential relaxation

$$
P\left(\gamma^{+}, t\right)=\left(1+\left(2 P_{0}-1\right) \exp (-t / N)\right) / 2
$$

where $P_{0}=P\left(\gamma^{+}, 0\right)$. This result leads to an exponential decay in the local fields, $\phi_{i}(t) \propto \exp (-t / N)$. As a result, the system evolves towards a (critical) state where the sum of the inputs introduced by the coevolving partners are small and so small changes involving single connections can generate extinctions.

We can use the entropy of connections per species, i. e. the Boltzmann entropy

$$
H\left(P\left(\gamma^{+}, t\right)\right)=-P\left(\gamma^{+}, t\right) \log \left(P\left(\gamma^{+}, t\right)\right)-\left(1-P\left(\gamma^{+}, t\right)\right) \log \left(1-P\left(\gamma^{+}, t\right)\right)
$$

as a quantitative characterization of our dynamics. The Boltzmann entropy (also known as the Shannon entropy) gives us a measure of disorder but also a measure of uncertainty. It is bounded by the following limits: $0 \leq H\left(P\left(J^{+}, t\right)\right) \leq \log (2)$. These limits correspond to a completely uniform distribution of connections (i. e. $P\left(\gamma^{+}, t\right)=1$ and $\left.P\left(\gamma^{-}, t\right)=0\right)$ with zero entropy and to a random distribution with $P\left(\gamma^{ \pm}, t\right)=1 / 2$ which has the maximum entropy. Our rules make possible the evolution to the maximum network complexity, here characterized by the upper limit of the entropy.

As $H\left(P\left(\gamma^{+}, t\right)\right)$ grows, after a large extinction event, towards its maximum value $H^{*}=\log (2)$, sudden drops take place near large extinctions. So our system slowly evolves towards an "attractor" characterized by a randomly connected network. At such state, small changes of strength $1 / N$ can modify the sign of $\phi_{i}$ and extinction may take place. At this point, one clearly sees what is the role that external perturbations play: for them to trigger a large extinction, it is necessary that they act on a system located close to the critical state (here, the network close to the maximum entropy). A large extinction will never be found in a system with a low entropy of connections even with a reasonably large external perturbation. We can see that a wide distribution of extinctions is obtained: it is a power-law distribution, $N(s) \approx s^{-\tau}$ with $\tau=2.05 \pm 0.06$, consistent with the information available from the fossil record. Actually, the previous rules are able to reproduce the most relevant features of the observed dynamics in the fossil record, including the presence of well-defined transient trends (see table 2 and figure 10).

A mathematical model can be derived for this mean-field approximation 36]. The starting point is again a set of $N$ species, now characterized by a single integer quantity $\phi_{i}(i=1,2, \ldots, N)$. This quantity will play the role of the internal 
Table 2. some basic trends of macroevolutionary patterns. Observed and predicted by the SOC model (see text). All the quantitative reported exponents from the FR are reproduced by the SOC model as well as the qualitative features like the diversification curves. ((1): recent studies seem to suggest a lower exponent, of about $\gamma \approx 1.6[44]$ ).

\begin{tabular}{|c|c|c|}
\hline Property & Observed & SOC Model \\
\hline Dynamics & Punctuated & Punctuated \\
\hline Mass extinctions & Few events & Expected \\
\hline Diversity & Increasing & Transiently increasing \\
\hline Species decay & Exponential & Exponential \\
\hline Extinction pattern, $N(E)$ & Power law $(\alpha \approx 2)$ & Power-law $(\alpha \approx 2)$ \\
\hline Hurst exponent, $H$ & persistence, $H>1 / 2$ & persistence $H>1 / 2$ \\
\hline Genera lifetimes $N(T)$ & Power law ${ }^{(1)}(\gamma \approx 2)$ & Power law $(\gamma \approx 2)$ \\
\hline Genera-species $N_{g}(S)$ & Self-similar, $(\tau \approx 2)$ & Self-similar, $(\tau \approx 2)$ \\
\hline
\end{tabular}
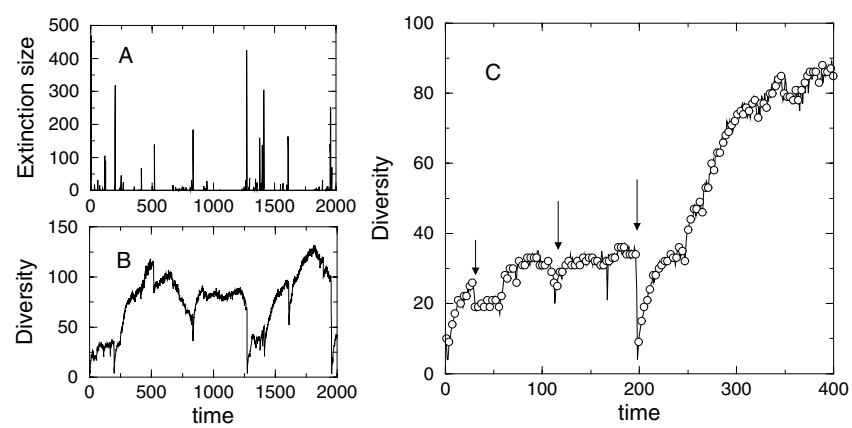

Fig. 10. Patterns of extinction and diversification for the mean field approximation to the network model. (A) Extinction dynamics, (B) diversity dynamics, here defined in terms of the number of genera in the system. In $(\mathrm{C})$ we show the transient increase in diversity. It fits quite well the observed trends displayed by the fossil record. We also show the occurrence of large extinctions, as indicated by the arrows.

field, as before. Each species is now represented by this single (now assumed integer) number $\phi_{i} \in\{-N,-N+1, \ldots,-1,0,1, \ldots, N-1, N\}$.

The dynamics consists of three steps: (a) with probability $P=1 / 2, \phi_{i} \rightarrow$ $\phi_{i}-1$, otherwise no change occurs (this is equivalent to the randomization rule in the network model); (b) all species with $\phi_{i}<\phi_{c}$ (below a given threshold) are extinct. Here we use $\phi_{c}=0$ but other choices give the same results. The number of extinct species, $0<E<N$, defines the extinction size. All $E$ extinct species are replaced by survivors. Specifically, for each extinct site (i. e. when $\phi_{j}<\phi_{c}$ ) we choose one of the $N-E$ survivors $\phi_{k}$ and $\phi_{j}=\phi_{k}$; (c) after an extinction event, a wide reorganization of the web structure occurs. In this simplified model this is introduced as a coherent shock. Each of the survivors are updated as $\phi_{k}=\phi_{k}+q(E)$, where $q(E)$ is a random integer between $-E$ and $+E$. 
The master equation for the dynamics involves the following three-step process:

$$
\begin{aligned}
N(\phi, t+1 / 3)= & \frac{1}{2} N(\phi, t)+\frac{1}{2} N(\phi+1, t) \\
N(\phi, t+2 / 3)= & N(\phi, t+1 / 3) \\
& +N(\phi, t+1 / 3) \sum_{m} \frac{m}{N-m} P(m)
\end{aligned}
$$

if $\phi>0$ and zero otherwise. Finally:

$$
\begin{aligned}
N(\phi, t+1)= & N(\phi, t+2 / 3)-N(\phi, t+1 / 3) \\
& +\sum_{q>-\phi} N(\phi+q, t+1 / 3) P(q)
\end{aligned}
$$

equations (19-21) lead to the full master equation for the dynamics:

$$
\begin{aligned}
N(\phi, t+1)= & \frac{1}{2} \sum_{q=-\infty}^{+\infty} \sum_{m} \frac{P(m)}{2 m+1} \theta(m-|q|) \\
& \times[N(\phi+q, t)-N(\phi+q+1, t)] \\
& +\frac{1}{2}[N(\phi, t)+N(\phi+1, t)] \sum_{m} \frac{m P(m)}{N-m}
\end{aligned}
$$

Where two basic statistical distributions, which are self-consistently related, have been used. These are:

$$
P^{*}(q)=\sum_{m} \frac{P_{e}(m)}{2 m+1} \theta(m-|q|)
$$

which is an exact equation giving the probability of having a shock of size $q$. Here $P_{e}(m)$ is the extinction probability for an event of size $m$, and now we have a mean-field approximation relating both distributions:

$$
P_{e}(m)=\sum_{q} P^{*}(q) \delta\left[\sum_{\phi=1}^{q-1} N(\phi)-m\right]
$$

The last equation uses the so called average profile $N(\phi)$. This function is the time-averaged distribution of $\phi$-values. Here we follow the Manrubia-Paczuski argument [36] for the mesoscopic regime $1 \gg q \gg N$. By Taylor-expanding the the master equation, i. e.

$$
\begin{aligned}
N(\phi)= & \frac{1}{2} \sum_{q=-\infty}^{+\infty} \sum_{m} \frac{P(m)}{2 m+1}\left\{2 N(\phi+q)+\left.\frac{\partial N}{\partial \phi}\right|_{\phi+q}+\left.\frac{1}{2} \frac{\partial^{2} N}{\partial \phi^{2}}\right|_{\phi+q}+\ldots\right\} \\
& +\frac{1}{2}\left\{2 N(\phi)+\left.\frac{\partial N}{\partial \phi}\right|_{\phi}+\left.\frac{1}{2} \frac{\partial^{2} N}{\partial \phi^{2}}\right|_{\phi}+\ldots\right\} \sum_{m} \frac{m P(m)}{N-m}
\end{aligned}
$$




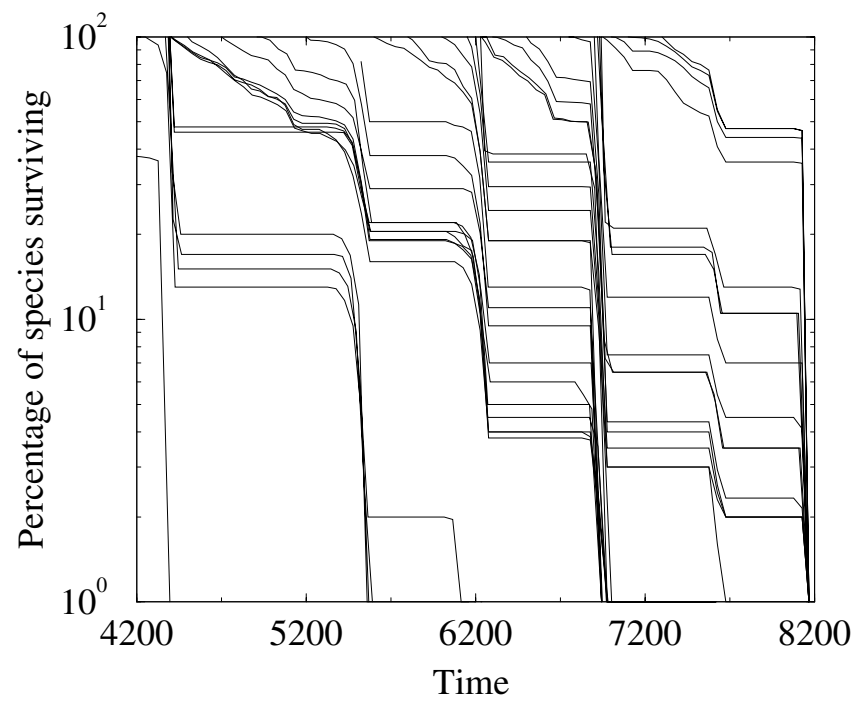

Fig. 11. Pattern of species extinction in the network model of macroevolution: on average, species get extinct in an exponential way, but this average pattern is punctuated by coextinction associated with mass extinction events.

and using a continuous approximation, it is easy to see that the previous equation reads:

$$
\begin{aligned}
& \frac{1}{2} \int d m \int_{-m}^{m} \frac{P(m)}{2 m}\left\{2\left[\frac{N(\phi+q)}{N(\phi)}-1\right]+\left.\frac{\partial L n N}{\partial \phi}\right|_{\phi+q}+\ldots\right\} \\
& +\frac{1}{2}\left\{2+\left.\frac{\partial L n N}{\partial \phi}\right|_{\phi}+\ldots\right\} \int \frac{m P(m)}{N-m} d m=0
\end{aligned}
$$

Using an exponential ansatz for the average profile, i. e. $N(\phi)=\exp (-c \phi / N)$, we can integrate each part of the last equation, assuming $N(\phi+q) / N(\phi)=$ $\exp (-c q / N) \approx 1-c q / N$. It is easy to check that the first term cancels exactly, the second gives $-2 c / N$ and the third scales as $(1-O(1 / N)) N^{1-\tau}$. So the previous equation leads to:

$$
-\frac{2 c}{N}+N^{1-\tau} G\left(1-O\left(\frac{1}{N}\right)\right)=0
$$

in order to satisfy this equality, we need $\tau \approx 2$, which gives us the scaling exponent for the extinction distribution. This is confirmed by numerical simulations, which give $\tau=2$.

This model (actually both of them) also display the observed exponential decay in species survival displayed by fossil record data. In figure 11 we show an example of our runs. This corresponds to the law of constant mean extinction rate, also known as Van Valen's law [68]. As mentioned in the introduction, this 
law maintains that the probability of extinction within any group remains essentially constant through time. This is a consequence of the Red Queen theory and an observational result. This is, however, an average: on average, extinction rates are constant but a close inspection of the decay curves shows both continuous and episodic decays. The sudden, episodic drops are often associated with mass extinctions and are usually assumed to be the result of external perturbations.

The episodic nature of the species decay is easily explained by our model. Though long periods of stasis and low extinction rates give a constant decay, the same intrinsic dynamics generates the episodes of extinction involving several (some times many) species. The survivorship curves shown in figure 11 are generated by starting at a given (arbitrary) time step in the simulation and following all the species present at this time step. The exponential decay in the number of survivors is closely related to the monotonous drift that the system experiences towards the extinction threshold, due to the constant change of connections to random values. As we can see (and this is rather typical) both constant and episodic decays are observed. We do not need to seek for a special external explanation for the episodic decay. Obviously, an external cause can trigger a large extinction event by altering the network dynamics at the critical state. Extinctions are an unavoidable outcome of network dynamics. Though some selection of connections is present after each extinction event, unpredictability is always present. A given species with a high "fitness" (defined in terms of the total input field it receives) can get extinct in a few steps due to an extinction avalanche propagating throughout the network.

\section{Extinction in layered networks}

The third class of models to be analysed here involves a very important aspect of real ecologies: the presence of different trophic levels. This is important not only because it adds an ingredient of realism to the topology of species interactions, but also because it has deep consequences on the dynamics. Real ecologies have some amount of hierarchical organization that can be described (on a first approximation) as a set of layers of interacting species. Such layers go from primary producers at the bottom to top predators in the upper floor. The number of layers is limited through both energetic and dynamic constraints [46. This is, again, an oversimplification, since real ecosystems are not fully hierarchical. Actually, they show small world topology [40].

It is well known that the ffects of perturbations on primary producers can have important (if not devastating) consequences on other parts of the food chain. Such effects can be direct: an example is provided by the $\mathrm{K} / \mathrm{T}$ event. Here the extinction pattern in marine habitats is entirely compatible with the effects of decreased food supply for higher trophic levels due to the collapse of phytoplankton productivity [2]. At the end-Cretaceous, primary productivity declined suddenly and considerably. The further decay of many species at higher trophic levels in oceanic plankton communities was mainly due to this decline. 
But cascade effects are also triggered by the removal of keystone species at any level in the web. Keystone species are specially relevant because of their large impact on the community dynamics, stability and composition. Their loss can cause extinctions to cascade throughout the system. An example provided by the fossil record is the extinction of megaherbivores (such as mastodon and mammoth) at the end-Pleistocene 45. These species, mainly large herbivores, were essentially invulnerable to non-human predation on adults. As a consequence, they attained high (saturating) densities. Their effects on vegetation patterns were huge (as it occurs today with elephants) and their loss had a catastrophic impact. As a very attractive prey to humans, their extermination brought extensive vegetational changes, eventually generating the concomitant disappearance of so many other vertebrates.

Such cascading effects have been reported in different field studies [9] [10], and their ecological effects have long been suspected for the major mass extinctions. They have been incorporated in a simple model ecosystem with layered structure by Amaral and Meyer [1].

The model considers $L$ levels $(l=0,1, \ldots, L-1)$ with $N$ niches per level (figure 12). We can indicate the presence or absence of species at the $i$-th niche in the $l$-th level by a binary variable $S_{i}(l)$. The bottom species, belonging to the $l=0$ layer, define the group of organisms which do not feed on others. From $l=1$ to $l=L-1$ the species of these layers feed on $k$ or less species on the lower $(l-1)$-level.

The rules are very simple: (a) a new species is created at each niche at a rate $\mu$. If $l>0$, then $k$ prey species are randomly chosen from the $(l-1)$-layer; (b) at a given rate $p$, species at the bottom layer are extinct. Then for species at $l>0$ layers, extinction takes place if no input links are present. Thus if $W(i, j ; l-1 \rightarrow l)$ indicates the connection between species $i \in l-1$ and species $j \in l$ (here this connection is either one or zero) then:

$$
S_{j}(l)=\Phi\left[\sum_{i=1}^{N} W(i, j ; l-1 \rightarrow l)\right]
$$

where now $\Phi(z)=1$ for positive $z$ and zero for $z=0$. Clearly, the link among connected species will be able to generate avalanches of coextinction through different layers. From numerical simulations, Amaral and Nunez showed that in fact the distribution of avalanches is a power law with an exponent -2 , consistent with the fossil record data.

An elegant analytic derivation of this result was obtained by Barbara Drossel [19]. Let us consider the $k=1$ case. In this specific situation, each species feeds only on one prey species at the lower layer. Several species on different layers will eventually feed on the same bottom species, and the trophic structure looks like a set of trees starting at a single species in the lower level. From rule (b) each time a bottom species is removed (with probability $p$ ) a whole tree is gone and thus the size distribution of extinction events must be identical to the tree size distribution. 


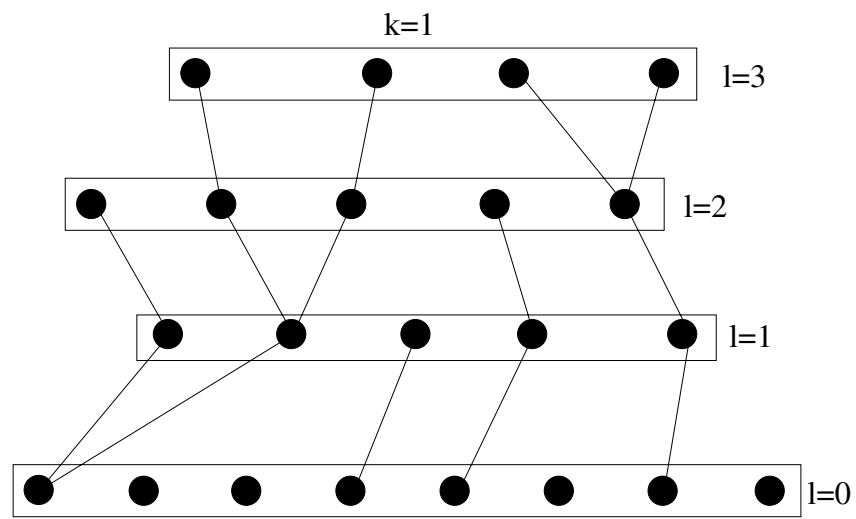

Fig. 12. Network structure of the simplest $(k=1)$ Amaral-Nunez layered ecosystem. The basal layer is formed by those species not feeding on others.

Assuming stationarity (and so a given mean number of species per level) a mean-field equation for the density of species at each layer $\rho_{l}$ can be derived:

$$
\frac{d \rho}{d t}=\mu(1-\rho)-p \rho_{l}
$$

This equation has a fixed point $\rho_{l}^{*}=\mu /(p+\mu)$. Let $\Lambda_{l}^{i}(t)$ the number of species at the $l$-th layer connected to the $i$-th bottom species. The growth of $\Lambda_{l}^{i}(t)$ follows the dynamical equation:

$$
\frac{d \Lambda_{l}^{i}}{d t}=\left[\frac{\mu\left(1-\rho_{l}\right)}{\rho_{l-1}}\right] \Lambda_{l-1}^{i}=p \Lambda_{l-1}^{i}
$$

And as a consequence the size of a whole tree $\mathcal{T}^{i}=\sum_{l} \Lambda_{l}^{i}$ will follow a linear equation

$$
\frac{d \mathcal{T}^{i}}{d t}=p \mathcal{T}^{i}
$$

i. e. we have

$$
\mathcal{T}^{i}(t)=\mathcal{T}^{i}(0) \exp (p t)
$$

with $\mathcal{T}^{i}(0)=1$. The size distribution of trees, $P(\Lambda)$ is linked to the age distribution $P(t)$ through $P(\Lambda)=P(t) d t / d \Lambda$. Here $P(t)$ follows the simple decay equation

$$
\frac{d P(t)}{d t}=-p P(t)
$$

and thus $P(t) \propto \exp (-p t)$. Using the previous results, we finally get the scaling relation:

$$
P(S) \propto S^{-2}
$$

in agreement with simulations. By analysing different sources of finite-size effects, Drossel shows that the previous results are robust for a broad range of parameter values. The previous analysis can be extended to the general $k>1$ problem. 
The previous proof involves the distribution of extinctions per dead species in the lowest level, let us call it $P_{d s}(s)$. One can also compute the distribution of extinction sizes per time step, $P(s)$, which is what the fossil record actually supplies, and adds all the extinctions of the first one along a time step [14. In this sense, it is worth to stress that the exponent -2 derived theoretically by Drossel is related but strictly is not the exponent of the extinction size distribution per time step, $P(s)$. In order to calculate this second distribution, one has to combine $P_{d s}(s)$ with the Poisson statistics, $P_{o}(l)$, for the number of extinctions in this level. Even if one neglects the correlations between one extinction and the next ones, the resulting expression, namely

$$
P(s)=\sum_{l=1}^{s} P_{o}(l) \sum_{\sum_{j=1}^{l} s_{j}=s} P_{d s}\left(s_{1}\right) \ldots P_{d s}\left(s_{l}\right),
$$

is very cumbersome to deal with analytically, so that, in practice, only numerical simulations can solve it. If one simulates a Poisson process with $P_{d s} \sim s^{-2}$ for the parameters used in our simulations, one finds the same scaling relation. Therefore, there is no contradiction between both distributions. In fact, as long as the average number of dead species per unit time in the lowest level is not high, if one removal gives rise to a big extinction, it is likely that this is the only big extinction taking place in that time step, since $P_{d s} \sim s^{-2}$ decreases rapidly with $s$. Therefore $P(s) \simeq P_{d s}(s) \sim s^{-2}$.

One can also estimate the location of the maximum in the extinction distribution, $P(s)$. We know that when one species in the lowest level dies, the probability for an extinction of size $s$ scales as $s^{-2}$. This means that $60 \%$ of the extinctions due to an extinction in the lowest level are of size 1 ( since $\left.\zeta^{-1}(2) \simeq 0.6\right)$. Then, most of the time a species in $l=1$ is gone with no further cascade. Now, if $N_{1}$ denotes the average number of species in the first level at the stationary state, this implies that most of the time the number of extinctions in a time step will be $\simeq N_{1} p$. It is easy to see that $N_{1} \approx N(1-p / \mu)$, so that for $N=1000, \mu=0.02$ and $p=0.01, N_{1} p \approx \% 5$, meanwhile for the case $\mu=0.004$ and $p=0.002$, one has $N_{1} p \approx \% 1$. These predictions for the location of the maxima agree well with numerical simulations, as shown in the last: the maximum is found at $s \sim 5$ in the first case, but it is absent in the second one (figure 13).

\section{Discussion}

It is generally agreed that species may go extinct because they are unable to evolve rapidly enough to meet changing circumstances or because their niches disappear. In the second case, no capacity for rapid evolution could save them from extinction. Theoretical models have been traditionally based on assumptions invoking either individual-based selection/adaptation mechanisms or externalities such as meteorite impacts. But in both cases the underlying ecological organization is essentially ignored. Species are effectively isolated entities whose extinction has little influence on ecosystem functioning (and thus in promoting 


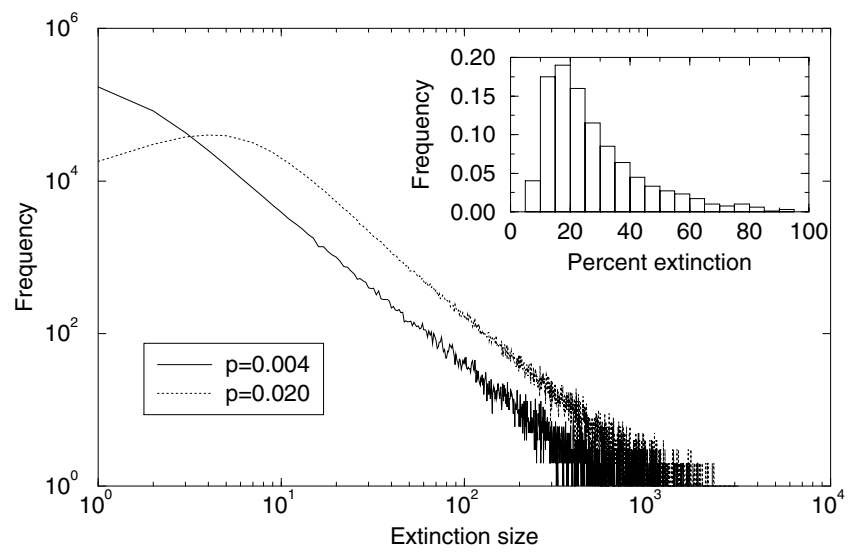

Fig. 13. Scaling in the Amaral-Meyer model. Here we show the distribution of extinction events for the AM model. The values of the parameters are: $\mu=0.02, p=0.01$, and $\mu=0.004, p=0.002$. A maximum is found in the first case, in agreement with the fossil record (see inset).

further extinctions). But these assumptions are far from true: the trophic nature of ecological interactions makes a big difference. When a species is gone, its effect can be very small or very large.

Biotic responses have been of great importance in the past [37 [38] as they are today in our biosphere. Keystone species are probably an inevitable result of ecological complexity [28] and in that sense their removal from an ecosystem can have highly nonlinear effects [66]. Such nonlinearity and the high-level patterns emerging from network dynamics are likely to decouple micro- from macroevolutionary processes. It is the global features of the food web what matters, not the specific properties of the species constituting the web. In Bak's sandpile metaphor, we cannot understand the sandpile behavior by looking at gravitation and friction acting on individual grains. Instead, we recognized that the sandpile at the critical state must be analysed as a collective phenomenon, since new properties (interactions among grains) are at work. Similarly, natural selection and adaptation are operating on species in complex ecologies, but the complete picture requires the consideration of interactions among species. In the long run, the effects of selection pressures on single species are like gravity and friction operating on grains of sand: we need to take them into account, but they cannot explain the avalanches.

The future of this area, in my view, will need to incorporate a detailed understanding of the information provided by the fossil record at different scales in space and time. Instead of considering a few sets of large-scale patterns, available information dealing with evolutionary responses by well-defined groups whould be taken into account. This is particularly important when looking at recovery patterns [21] [22] [23]. Recovery patterns provide a unique window to explore 
the structure and evolution of paleoecosystems. Ecological links do not fossilize, but the underlying structure of ancient food webs can be inferred from the fossil record. In this context, new models of evolution [67] exploring the responses of different trophic levels to mass extinctions events will be very useful not only in order to understand how the biosphere reacted in the past to external challenges, but also to provide useful insight into the current human-driven mass extinction event.

The previous models and others not reviewed here (see in particular the work by Caldarelli et al. 13]) are a first step towards a complete theory of large-scale evolution. These models are typically non-historic, and in that sense they ignore some essential ingredients of the true dynamics of biological entities. But they are able to capture some large-scale trends that other, more detailed approximations cannot provide without an important loss of real understanding. In spite of their simplicity, they clearly indicate that such a theory is feasible, if we are able to go beyond the infinite details provided by the fossil record. As Niles Eldredge said: "...we should not despair. There is real order in all this apparent chaos. Life has had a long and complex, but ultimately comprehensible, history. There are patterns repeated over and over again as new species come and go, and as ecosystems form and fall apart. These organizing principles of life's history are the processes of evolution".

\section{Acknowledgements}

The auhor thanks S. Kauffman, G. Eble, S. Manrubia and J. M. Montoya for many discussions on evolutionary ecology. This work has been supported by a grant CICYT PB97-0693 and The Santa Fe Institute (RVS).

\section{References}

1. Amaral, L. A. N. and Meyer, M. Phys. Rev. Lett. 82, 652 (1999)

2. Arthur, M. A., Zachos, J. C. and Jones, D. S. Cretaceous Research 8, 43 (1987)

3. Bak, P., Tang, C. and Wiesenfeld, K. Phys. Rev. Lett. 59, 381 (1987)

4. Bak, P., Flyvbjerg, H. and Lautrup, B. Phys. Rev. A 46, 6724 (1992)

5. Bak, P. and Sneppen, K. Phys. Rev. Lett. 714083 (1993)

6. Bak, P.How Nature Works, Springer (1996)

7. Benton, M. J. Science 268, 52 (1995)

8. Benton, M.J. In: Palaeobiology (ed. D.E.G. Briggs \& P. R. Crowther). Blackwells. Oxford (1995)

9. Brown, J. H. and Heske, E. J. Science, 250, 1705 (1991)

10. Brown, J. H., in Complexity: Metaphors, Models and Reality Cowan, G., Pines, D., and Meltzer, D., eds., pp. 419-449, Addison Wesley (1994)

11. Burlando, B. J. theor. Biol. 146, 99 (1990)

12. Burlando, B. J. theor. Biol. 163, 161 (1993)

13. Caldarelli, G., Higgs, P. G. and McKane, A. J. J. Theor. Biol. 193, 345 (1998)

14. Camacho, J. and Solé, R. V. Phys. Rev. E 62, 1119-1123 (2000)

15. Chaloner, W. and Hallam, A. (eds) Phil. Trans. R. Soc. London B325, 239-488 (1989) 
16. Conway Morris, S. Phil Trans. Roy. Soc. Lond. B 353, 327 (1998)

17. Conway Morris, S. The Crucible of Creation. Oxford U. Press (1998)

18. Dimri, V. P. and Prakash, M. R. Earth and Planetary Science Letters (to appear)

19. Drossel, B. Phys. Rev Lett. 81, 5011 (1998)

20. Droser, M. L., Bottjer, D. J. and Sheehan, P. M. Geology 25, 167 (1997)

21. Erwin, D. H. The Great Paleozoic Crisis: Life and Death in the Permian. Columbia U. Press (1993)

22. Erwin, D. H. Nature 367, 231 (1994)

23. Erwin, D. H. Trends Ecol. Evol. 13, 344 (1998)

24. Hewzulla, D., Boulter, M. C., Benton, M. J. and Halley, J. M. Proc. Roy. Soc. London B 354, 463 (1999)

25. Jablonski, D. Science 284, 2114 (1999)

26. Jablonski, D., Erwin, D. H. and Lipps, J. H. 1996. Evolutionary paleobiology. Chicago U. Press

27. Jablonski, D. Paleobiology Suppl. 26, 15 (2000)

28. Jain, S. and Krishna, S. preprint arXiv:nlin.AO/0005039 (2000)

29. Kauffman, S. and Johnsen, J. J. Theor. Biol. 149, 467 (1991)

30. Kauffman, S. The Origins of Order, Oxford U. Press, Oxford (1993)

31. Kauffman, S. At Home in the Universe, Oxford U. Press, Oxford (1997)

32. Kirchner, J. W. and Weil, A. Nature 395, 337 (1998)

33. Kirchner, J. W. and Weil, A. Nature 404, 177 (2000)

34. Korvin, G. 1992. Fractal Models in the Earth Sciences, Elsevier

35. Maddox, J. Nature 371, 197 (1994)

36. Manrubia, S. C. and Paczuski, M. Int. J. Mod. Phys. C 9, 1025 (1998)

37. Maynard Smith, J. Phil. Trans. R. Soc. Lond. B 325, 241 (1989)

38. McKinney, M. L. and Drake, J. A. (eds.) Biodiversity Dynamics, Columbia U. Press (1998)

39. Miller, A. I. Paleobiology Suppl. 26, 53 (2000)

40. Montoya, J. M. and Solé, R. V. Santa Fe Institute Working Paper 00-10-059 (2000)

41. Newman, M. E. J. Proc. Roy. Soc. London B 263, 1605 (1996)

42. Newman, M. E. J. J. Theor. Biol. 189, 235 (1997)

43. Newman, M. E. J. and Eble, G. Paleobiology 25, 434 (1999)

44. Newman, M. E. J. and Palmer, R. Santa Fe Institute Working Paper 99-08-061 (1999)

45. Owen-Smith, N. Paleobiology 13, 351 (1987)

46. Pimm. S. The Balance of Nature, Chicago Press (1991)

47. Plotnick, R. E. and McKinney, M. Palaios 8, 202 (1993)

48. Plotnick, R. E. and Sepkoski. J. J. Paleobiology 27, 126 (2001)

49. Rao, R. M. and Bopardikar, A. S. Wavelet Transforms : Introduction to Theory and Applications, Addison-Wesley (1998)

50. Rampino, M. R. and Sothers, R. B. Nature 308, 709 (1984)

51. Rampino, M. R. and Sothers, R. B. Earth, Moon and Planets 72, 441 (1995)

52. Raup, D. Extinctions: Bad Genes or Bad Luck? Oxford U. Press (1993)

53. Raup, D. M. and Sepkoski, J. J., Jr. Proc. Natl. Acad. Sci. USA 81, 801 (1984)

54. Raup, D. M. Science 231, 1528 (1986)

55. Rowe, G. W. Theoretical Models in Biology, Oxford U. Press (1994)

56. Sepkoski, J. J., Jr. Paleobiology 10, 246 (1984)

57. Sibani, P. Phys. Rev. Lett. 79, 1413 (1997)

58. Sneppen, K. et al. Procs. Natl. Acad. Sci. USA 92, 5209 (1995)

59. Solé, R. V. Complexity 1, 40 (1996) 
60. Solé, R. V., Bascompte, J. and Manrubia, S. C. Proc. Roy. Soc. London B 263, 1407 (1996)

61. Solé, R. V. and Manrubia, S. C. Phys. Rev. E 54 R42 (1996)

62. Solé, R. V. and Bascompte, J. Proc. R. Soc. London 263, 161 (1996)

63. Solé, R. V., Manrubia, S. C., Benton, M. and Bak, P. Nature 388, 764 (1997)

64. Solé, R. V., Manrubia, S. C., Kauffman, S. A., Benton, M. and Bak, P. Trends in Ecol. 14, 156 (1999)

65. Solé, R. V., Manrubia, S. C., Pérez-Mercader, J., Benton, M. and Bak, P. Adv. Complex Syst. 1, 255 (1998)

66. Solé, R. V and Montoya, J. M. Santa Fe Institute Working Paper 00-10-060 (2000)

67. Solé, R. V., Montoya, J. M. and Erwin, D. H. Phil. Trans. Roy. Soc. London B. (to appear)

68. Van Valen, L. Evol. Theory 1, 1 (1973) 